\title{
Salivary Alpha-Amylase Mediates the Increase in Hunger Levels in Adolescents with Excess Weight after Viewing Food Images
}

\author{
María Moreno-Padilla, PhD,' Enrique F. Maldonado-Montero, PhD, \\ Alfredo Enguix-Armada, $\mathrm{PhD}^{3}$, and Gustavo A. Reyes del Paso, $\mathrm{PhD}^{1}$
}

\section{Abstract}

Background: Salivary alpha-amylase (sAA) initiates the digestion process in the mouth and its levels might influence feelings of hunger and the propensity toward obesity. This study aims to evaluate basal differences in sAA between adolescents with excess weight (EW) and normal weight (NW), and the associations between sAA levels and feelings of hunger after viewing food images.

Methods: Adolescents (13-18 years old) classified as EW $(n=30)$ or NW $(n=30)$ participated in the study. Saliva samples were collected before the administration of a food-choice task. Hunger was evaluated before and after the food-choice task.

Results: EW adolescents showed lower basal sAA levels than NW adolescents and a greater increase in hunger levels after viewing food images. In addition, sAA levels had a significant inverse relationship with the increase in hunger in EW adolescents, but not in NW adolescents. Finally, significant inverse associations between sAA, BMI, and body fat percentage were found.

Conclusions: Levels of hunger and changes therein, after viewing food are dependent on sAA levels in EW adolescents. This finding indicates that sAA levels may be a mediator of feelings of hunger in individuals with overweight in the context of viewing food cues, suggesting the utility of the sAA enzyme as a marker of hunger and propensity toward obesity.

Keywords: adolescents; food images; hunger; obesity; salivary alpha-amylase

\section{Introduction}

$\mathbf{O}$ besity is one of the most common chronic health risk factors; its prevalence continues to rise rapidly in children and adolescents, and it leads to several long-term health complications. ${ }^{1,2}$ Adolescence is a particularly high-risk period for weight gain because of the interaction of natural metabolic changes with behavioral risk factors. ${ }^{3}$ Weight gain during adolescence is a major risk factor for adult obesity and metabolic syndrome. ${ }^{4}$ Our "obesogenic" food environment is characterized by a plentiful supply of appetizing, high-energy, and low-priced food that is constantly accessible and aggressively promoted.,5 Maintaining a healthy weight has become a difficult challenge in such an environment.

The processes regulating eating behavior are complex and comprise chemical, sensory, behavioral, homeostatic, and hedonic mechanisms, controlled by hunger and satiety signals in peripheral and central pathways. ${ }^{7-9}$ Regarding endocrine systems, some peptides and proteins, like leptin, insulin, ghrelin, and peptide YY provide information about metabolic physiology, and can therefore be used as biomarkers of eating behavior regulation and obesity. ${ }^{10,11}$

One such potential biomarker of eating behavior regulation is salivary alpha-amylase (sAA). ${ }^{12-14}$ Alpha-amylase, an enzyme present in many tissues, hydrolyses bonds of large polysaccharides, like glycogen and starch, yielding larger amounts of small molecules such as maltose and glucose. Alpha-amylase is found in saliva (i.e., ptyalin) and it starts the food digestion process in the mouth by breaking polysaccharides into progressively more simple soluble molecules (erythrodextrin, achrodextrin, and amylodextrin) to ultimately produce maltose and glucose. ${ }^{15}$ Levels of sAA, through its influence on carbohydrate metabolism and digestion, may influence hunger levels and obesity predisposition.

\footnotetext{
'Department of Psychology, University of Jaén, Jaén, Spain.

2Department of Psychobiology and Methodology of Behavioral Sciences, University of Málaga, Málaga, Spain.

${ }^{3}$ Department of Clinical Analysis, Virgen de la Victoria Hospital, Malaga, Spain.
} 
There are polymorphisms present in the human genome encoding for SAA, in which between-subject differences in the number of copies can be observed. The number of gene copies is positively associated with levels of sAA. ${ }^{16}$ A reduced number of copies in the sAA gene is associated with increased adiposity, BMI, obesity risk, ${ }^{17}$ and metabolic abnormalities ${ }^{18,19}$ and with reduced preabsorptive insulin release. $^{20}$

Furthermore, recent studies have confirmed that in a food intake-related context, higher sAA levels are associated with a physiological measure of perceived satiety. ${ }^{21-24}$ In individuals with lower sAA levels, the digestion of carbohydrates will be slow, which would serve to maintain high levels of hunger over a longer period, leading to greater food consumption before achieving satiety. In the long term, this would increase the propensity toward obesity. BMI has also been found to be negatively correlated with average morning sAA levels. ${ }^{25}$

This study aims to evaluate basal sAA differences between excess weight (EW) and normal weight (NW) adolescents, and the association between sAA and subjective hunger levels after viewing food images. Most studies have focused on the role of sAA as a biomarker of hunger after food consumption ${ }^{21-24}$; however, to date, very few studies have analyzed the association between sAA levels and feelings of hunger in a food-cue display context. Furthermore, no research has focused on evaluating sAA in adolescents with EW, and most recent studies have been focused on the number of copies of the sAA gene, with few directly comparing sAA levels between groups differing in body weight. ${ }^{16}$ Given that lower sAA levels slow down the digestion of carbohydrates, low-glucose (pre-eating) levels would be maintained for longer, thus stimulating further eating. In this context, and according to the previous evidence, we hypothesize that sAA levels will be lower in excess than in NW adolescents and that levels of hunger, and changes therein, during the viewing of food images will be associated with sAA levels, with lower sAA being related to greater feelings of hunger in both groups.

\section{Methods}

\section{Participants}

The participants were 60 adolescents ( 25 male and 35 female participants) recruited between September and February 2017 from high schools in Jaén, Spain. They were all 13-18 years of age, and were selected based on their age-adjusted BMI percentile following the guidelines of the International Obesity Task Force criteria. ${ }^{26}$ Adolescents with NW $(n=30)$ had age-adjusted BMI values between the 5th and 84th percentiles, and adolescents with EW $(n=30)$ had age-adjusted BMI values above the 85th percentile. Age, sex, BMI, and body fat percentage data are given in Table 1. The inclusion criteria were as follows: (1) age between 13 and 18 years and (2) no history of neurological, psychiatric, or eating disorders. All participants had normal or corrected to normal vision. This study was

\begin{tabular}{|c|c|c|c|c|c|c|}
\hline & \multicolumn{2}{|c|}{$\begin{array}{l}\text { Excess } \\
\text { weight }\end{array}$} & \multicolumn{2}{|c|}{$\begin{array}{c}\text { Normal } \\
\text { weight }\end{array}$} & \multirow{2}{*}{$\begin{array}{c}t^{a} l \\
\text { Chi-square }\end{array}$} & \multirow[b]{2}{*}{$p$} \\
\hline & Mean & SD & Mean & SD & & \\
\hline Age & 15.38 & 1.76 & 15.42 & 1.37 & $-0.08^{a}$ & 0.935 \\
\hline $\begin{array}{l}\text { Sex } \\
\text { (\%men/women) }\end{array}$ & \multicolumn{2}{|c|}{$46.7 / 53.3$} & \multicolumn{2}{|c|}{$36.7 / 63.3$} & $0.62^{\mathrm{b}}$ & 0.432 \\
\hline BMI & 28.53 & 2.97 & 20.05 & 2.05 & $12.87^{\mathrm{a}}$ & $<0.001$ \\
\hline$\%$ Body fat & 28.05 & 7.78 & 17.32 & 7.72 & $5.36^{\mathrm{a}}$ & $<0.001$ \\
\hline
\end{tabular}

approved by the Ethics Committee of the Universidad de Jaén and the procedures followed were in accordance with the Declaration of Helsinki, 1975, as revised in 2008.

\section{Saliva Sampling and SAA Analysis}

The participants were instructed not to smoke, eat, or drink coffee for at least 1 hour before the experiment. All the experimental sessions were conducted at the same time of the day (4-5 pm). Saliva samples were collected by spitting directly into disposable glass-capped tubes $(75 \times 12 \mathrm{~mm})$ set in a small polystyrene container suitable for storage in a freezer compartment. ${ }^{27,28}$ All tubes were labeled and dated after sample collection and stored at $-20^{\circ} \mathrm{C}$ until analysis.

Saliva samples were diluted at 1:500 in bi-distil water after each sample was defrosted once. The sAA levels were analyzed by an enzymatic colorimetric assay using a Dimension Vista system. ${ }^{29}$ Previously, two saliva pools were created with high and low concentrations of sAA activity; these were used as controls for each assay. The intra- and interassay coefficients of variability for sAA levels were $<10 \%$ in our analysis. The obtained values are markers of enzymatic sAA levels in saliva (arbitrary units; U/mL). ${ }^{30}$

\section{Food-Choice Task}

A food preference task was used in this study. Two types of food pictures were used: appetizing (high levels of fats and/or sugars) and healthy. All pictures were acquired ad hoc for the study under standardized presentation and lighting conditions. Therefore, all images were matched for visual properties (size, brightness, clarity, and contrast) and size ( $\sim 1$ standardized portion for all images). The appetizing food images included sausages and chocolate, whereas fruits and salads were presented among the healthy food images. In each trial, pairs of these different types of food images were presented according to three conditions (appetizing vs. healthy, appetizing vs. appetizing, and healthy vs. healthy). Participants had to choose between the two possibilities on each trial by pressing a key on 
a computer keyboard. The total duration of the task was $\sim 5$ minutes. Each trial began with a fixation cross, presented for 3-6 seconds (i.e., varying among trials). Then, the two food images appeared side-by-side on the screen for 5 seconds, with the positions of the appetizing and healthy foods (left or right side) varying among trials. The order of presentation of the pictures was counterbalanced across the adolescents. Subsequently, the fixation cross was presented again. The task was composed of a total of 30 choice trials ( 10 elections for each condition) preceded by four training trials.

\section{Procedure}

Figure 1 provides a schematic representation of the experiment. After obtaining permission from the high school's directors, the study was described to individual classes of students and their participation was requested. Students who were interested in taking part sent us a signed informed consent form (signed by their parents if they were minors). Then, the participants were assigned to an experimental group, and a specific day on which to complete the experimental session. Sessions started at $4 \mathrm{pm}$, and participants were required to be satiated (having had lunch about 1 hour before) and to not have taken any caffeine. Weight and height were self-reported by participants for recruitment purposes, and BMI was calculated in the laboratory using the height and weight data collected on arrival. Body composition parameters were also measured, using the Bodystat ${ }^{\circledR} 1500$ monitoring unit. Subsequently, saliva samples were acquired over $\sim 5$-minute period. Then, participants performed the food-choice task. A visual analog scale (VAS) was used to rate subjective hunger levels, on a scale ranging from 1 (not hungry) to 10 (very hungry). The VAS was completed before (pretask) and after completion of the food-choice task (post-task). The total duration of the study was $\sim 1$ hour. This study formed part of a larger investigation of the effects of hunger levels on risk taking in adolescents. A more detailed description of the study procedure can be found elsewhere. ${ }^{31}$

\section{Statistical Analyses}

No deviation from normality or homogeneity of variance was observed in the hunger and sAA level data (all $p$-values $<0.05$ on both the Kolmogorov-Smirnov and Levene tests). Group comparisons were carried out with Student's $t$-test for independent samples. Hunger levels were analyzed by repeated-measures analysis of variance with time (pre- and post-task) as the repeated-measure factor and group (EW vs. NW) as the between-subject factor. In addition, analysis of covariance (ANCOVA) was performed including the covariable sAA, to assess its influence on hunger change, prevs. post-task. Correlations between variables were analyzed by Pearson's product-moment correlation coefficient. To simplify the analyses, hunger change scores were calculated as the post-task value minus the pretask value.

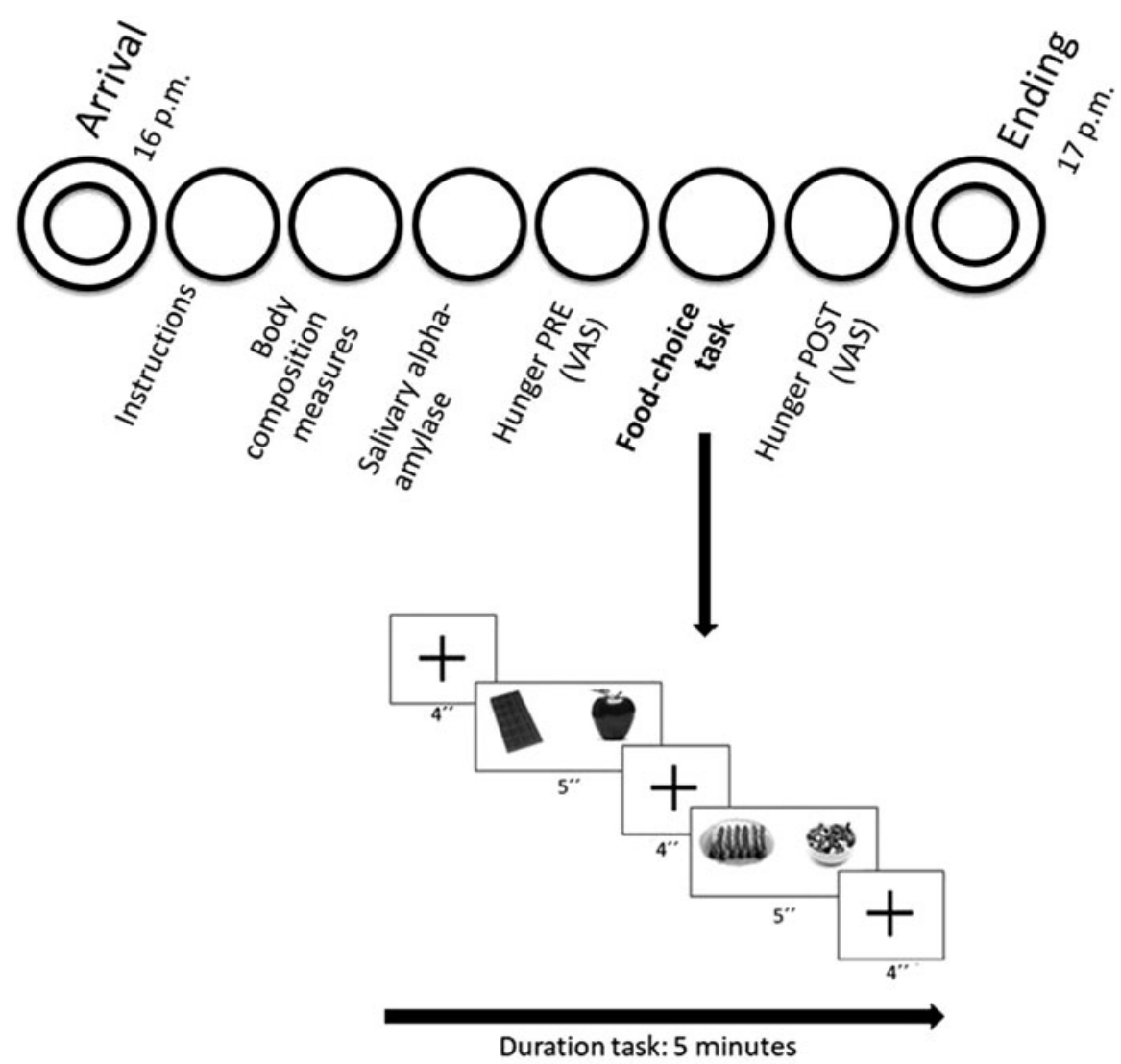

Figure 1. Schematic representation of the experiment. 


\section{Results}

Group differences were found in sAA levels $(t=-3.13$, $p=0.003, \delta=-0.82$ ), with adolescents with EW displaying lower sAA levels than the NW adolescents (Fig. 2). Group differences in hunger levels did not reach significance in either the pretask $(t=1.75, p=0.086, \delta=0.45)$ or post-task $(t=-1.94, p=0.057, \delta=-0.5)$ evaluation (Fig. 3). However, a time $\times$ group interaction was found for VAS hunger scores $\left(F(1,58)=10.82, p=0.002, \eta_{p}^{2}=0.16\right)$. Although both groups showed a significant increase in hunger levels between the pre- and post-task evaluation, the increase was greater in the EW adolescents $(F(1,29)=42.05, p<0.001$, $\left.\eta_{p}^{2}=0.59\right)$ than in the NW adolescents $(F(1,29)=16.13$, $p<0.001, \eta_{p}^{2}=0.36$ ) (Fig. 3).

The ANCOVA revealed a $\mathrm{sAA} \times$ group $\times$ time interaction $\left(F(1,55)=4.64, p=0.014, \eta_{p}^{2}=0.14\right)$. Levels of sAA had a significant effect on the change in hunger levels in the EW group $\left(F(1,27)=8.39, p=0.007, \eta_{p}^{2}=0.24\right)$, but not in the NW group $\left(F(1,27)=1.24, p=0.28, \eta_{p}^{2}=0.04\right)$. Furthermore, in the EW group levels of sAA were inversely correlated with post-task hunger levels $(r=-0.45, p=0.014)$ and the change in hunger levels $(r=-0.49, p=0.007)$ (Fig. 4). However, in the NW group no associations were found between sAA and post-task hunger levels $(r=0.15$, $p=0.434)$ and the change in hunger levels $(r=0.21$, $p=0.276$ ). Finally, among the whole sample, levels of sAA were inversely correlated with body fat percentage $(r=-0.27, p=0.04)$ and BMI $(r=-0.38, p=0.003)$.

\section{Discussion}

Our results showed that adolescents with EW had lower basal sAA levels than NW adolescents. Furthermore, adolescents with EW displayed a greater increase in hunger levels (VAS scores) after viewing food images task than the NW adolescents. In addition, sAA levels had a significant inverse association with the change in hunger level in adolescents with EW, but not in NW adolescents. As expected, levels of hunger and changes therein during the viewing of food images were dependent on sAA levels, with lower sAA being associated with greater feelings of hunger. However, this was observed only in adolescents with EW. Finally,

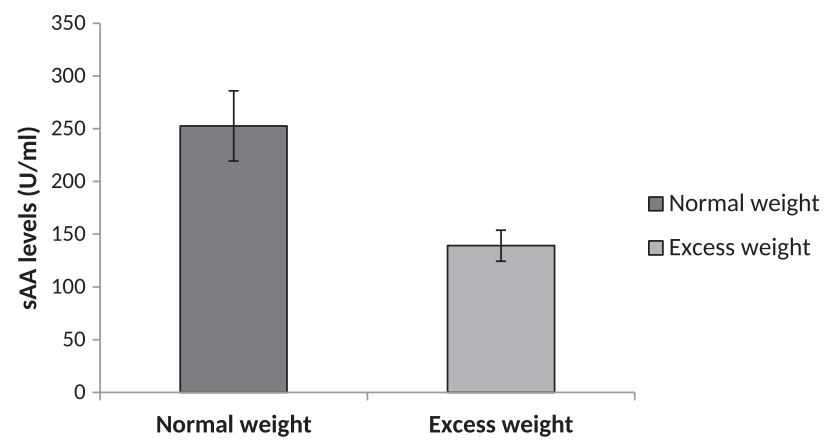

Figure 2. Group differences in sAA concentrations $(\mathrm{U} / \mathrm{mL})$. SAA, salivary alpha-amylase.

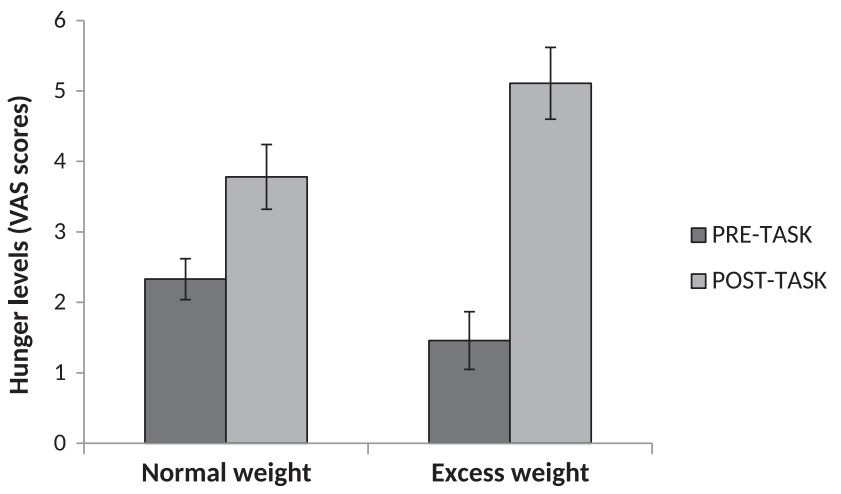

Figure 3. Group differences in hunger levels (VAS scores) from the pre- to post-task evaluation. VAS, visual analog scale.

among the whole sample, significant inverse associations between sAA, BMI, and body fat percentage were found.

These results corroborate previous findings suggesting a negative association between BMI and sAA levels. Reduced copy numbers of the gene encoding for sAA have been reported in people with obesity, ${ }^{16-18}$ with evidence of an inverse association between enzymatic sAA levels and BMI emerging over a 9 -year follow-up period ${ }^{16}$ implying a possibly causative effect of sAA levels on decreased adiposity. However, until now no research had compared sAA levels between EW and NW adolescents.

Given the function of sAA in carbohydrate catalysis, increased sAA levels are associated with more rapid and effective carbohydrate digestion. Therefore, the serum glucose level rises more quickly and chemical satiety signals are generated earlier. Accordingly, sAA has been considered as a potential measure of satiety in food-related contexts. $^{21-23}$ Chemical satiety signals include peptides,

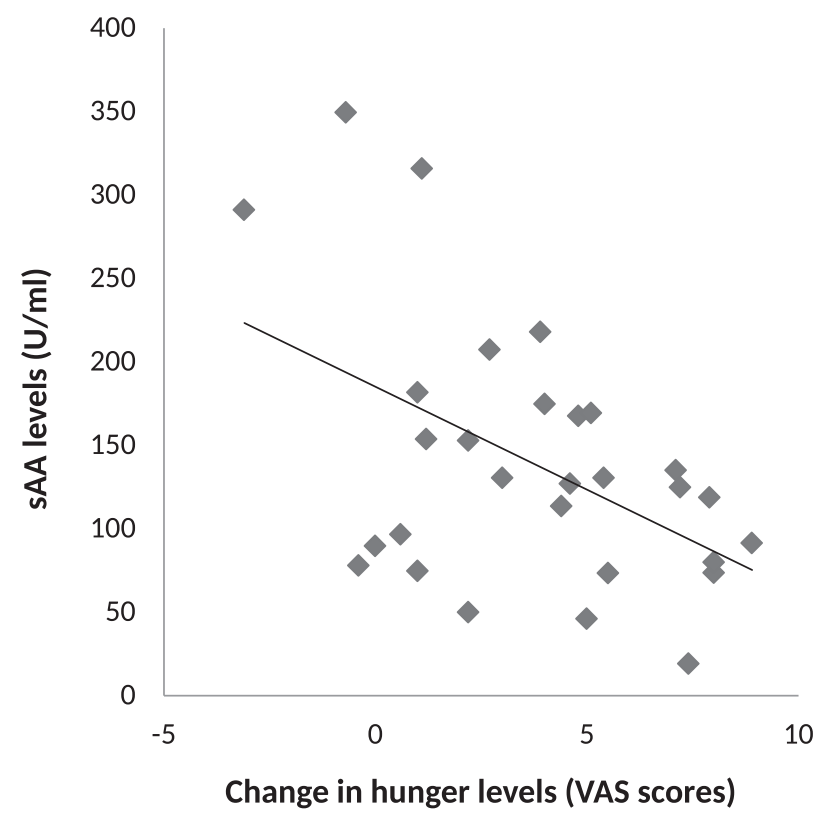

Figure 4. Scatterplot and regression line for the relationship between sAA levels (U/mL) and change in hunger levels (VAS scores) in excess weight adolescents. 
such as cholecystokinin, glucagon-like peptide 1, and peptide YY. These signals travel from the stomach to the brain; specifically, the hypothalamus receives and integrates this information to stimulate both satisfaction and satiety, thus controlling the duration of food intake episodes (i.e., volume of food ingested). ${ }^{32}$ In individuals with lower sAA levels, the catalysis of carbohydrates will be slower, such that the low predigestion levels of glucose are maintained for long, thereby favoring greater food consumption. In the long term, these lower sAA levels synergize with learning and the consolidation of habitual eating patterns, thus potentially leading to obesity.

Our results are coherent with previous studies suggesting an association between sAA and perceived satiety in food-related contexts. ${ }^{21-23}$ Harthoorn $^{23}$ reported that sAA systematically increased upon food consumption and satiation, and therefore serves as a potential measure of satiety and subsequent food intake. Our results showed that sAA was negatively associated with both the change (increase) in hunger and hunger feelings after the foodvisualization task, but not with hunger levels before the task. Furthermore, sAA levels were associated with the change in feelings of hunger in adolescents with $\mathrm{EW}$, but not in adolescents with NW. This finding could be explained by the fact that individuals with EW maintain low glucose levels for long because of lower sAA levels, which favors an increase in feelings of hunger, and thus potential subsequent overeating. Furthermore, the literature shows that adolescents with overweight and obesity are more sensitive to food cues, and are thus more likely to respond to them..$^{33-36}$

Previous studies have reported a positive association between sAA levels and feelings of satiation after food consumption. ${ }^{21-23}$ Our results showed a negative association between sAA levels and feelings of hunger after, but not before, viewing the food images in adolescents with EW. Therefore, our findings also indicate the special relevance of sAA to feelings of hunger evoked by viewing food images. Because these associations, and the mediating effect of sAA, were seen only in the adolescents with EW, sAA levels may influence the feelings of hunger and eating behaviors evoked by external cues in this population specifically. These results are innovative, where no previous study has explored the role of sAA in the context of viewing food images, nor its potential mediating effect on feelings of hunger.

Alpha amylase parameters have been shown to be associated with diabetes and metabolic abnormalities. Furthermore, a low serum amylase level was identified in diabetic patients (mainly those with insulin-dependent diabetes; type 1 diabetes) ${ }^{37,38}$ Another relevant mechanisms mediating sAA levels in adolescents with EW may be the development of insulin resistance. Obesity can induce insulin resistance, where the resulting increased insulin levels elicit dose-dependent stimulation of the sympathetic nervous system (SNS). ${ }^{39}$ The greater SNS activity and related increase in noradrenaline levels stimulate $\beta$-adrenergic receptors of the salivary parotid glands, in turn increas- ing sAA secretion. ${ }^{40}$ Therefore, this mechanism would likely increase (not decrease) sAA levels in adolescents with EW.

Regarding the limitations of this study, there are a number of issues that need to be addressed in future studies. These include the inclusion of fasted and nonfasted conditions, differentiation between participants with obesity, overweight, and NW, and the inclusion of adult participants. One important improvement could be the inclusion of a second measure of sAA after the food image task, to better evaluate the effect of the task on sAA levels and the influence of the sAA on the change in feelings of hunger. Furthermore, future studies should evaluate the longitudinal effects of sAA levels on weight gain. The lack of a control group in this study makes it difficult to discern whether the changes in feelings of hunger were the result of the viewing of food cues or the mere passage of time. Future studies including an appropriate control group will be necessary to address this limitation. Finally, although the results of performed ANCOVA suggest a causal role of sAA, we do not certainly know whether the reduced sAA levels in EW adolescents are a cause, a consequence, or simply a marker of high BMI. Future studies using other methodological designs should address this issue.

In summary, the results of this study showed that adolescents with EW experienced a greater increase in feelings of hunger after viewing food images, and that the magnitude of this increase was inversely associated with basal sAA levels. In fact, sAA levels concentrations could have influenced the change in hunger after viewing food images in adolescents with EW. This finding indicates that sAA levels might be a mediator of feelings of hunger in the context of viewing food cues in people with overweight, in turn suggesting the utility of the sAA enzyme as a marker of hunger levels, eating behavior, and obesity.

\section{Authors' Contributions}

M.M.-P. and G.A.R.d.P. designed the study. M.M.-P. carried out the experiment and analyzed the data. All authors were involved in writing the article and gave final approval to the submitted and published versions.

\section{Funding Information}

This work has not received any funding.

\section{Author Disclosure statement}

No competing financial interests exist.

\section{References}

1. Kimm SY, Obarzanek E. Childhood obesity: A new pandemic of the new millennium. Pediatrics 2002;110:1003-1007.

2. Sahoo K, Sahoo B, Choudhury AK, et al. Childhood obesity: Causes and consequences. J Family Med Prim Care 2015;4:187-192. 
3. Jasik CB, Lustig RH. Adolescent obesity and puberty: The "perfect storm." Ann N Y Acad Sci 2008;1135:265-279.

4. Must A, Naumova EN, Phillips SM, et al. Childhood overweight and maturational timing in the development of adult overweight and fatness: The Newton Girls Study and its follow-up. Pediatrics 2005;116:620-627.

5. Hill JO, Peters JC. Environmental contributions to the obesity epidemic. Science 1998;280:1371-1374.

6. Wadden TA, Brownell KD, Foster GD. Obesity: Responding to the global epidemic. J Consult Clin Psychol 2002;70:510-525.

7. Sáinz N, Barrenetxe J, Moreno-Aliaga MJ, et al. Leptin resistance and diet-induced obesity: Central and peripheral actions of leptin. Metabolism 2015;64:35-46.

8. Berthoud HR, Münzberg H, Morrison CD. Blaming the brain for obesity: Integration of hedonic and homeostatic mechanisms. Gastroenterology 2017;152:1728-1738.

9. Moreno-Padilla M, Verdejo-Román J, Fernández-Serrano MJ, et al. Increased food choice-evoked brain activation in adolescents with excess weight: Relationship with subjective craving and behavior. Appetite 2018;131:7-13.

10. De Graaf C, Blom WAM, Smee et al. Biomarkers of satiation and satiety. Am J Clin Nutr 2004;79:946-961.

11. Meier U, Gressner AM. Endocrine regulation of energy metabolism: Review of pathobiochemical and clinical chemical aspects of leptin, ghrelin, adiponectin, and resistin. Clin Chem 2004;50:1511-1525.

12. Marrelli M, Loizzo MR, Nicoletti M, et al. Inhibition of key enzymes linked to obesity by preparations from Mediterranean dietary plants: Effects on $\alpha$-amylase and pancreatic lipase activities. Plant Foods Hum Nutr 2013;68:340-346.

13. Granger DA, Kivlighan KT, El-Sheikh M, et al. Salivary sAA in biobehavioral research: Recent developments and applications. Ann N Y Acad Sci 2007;1098:122-144.

14. Aydin S. A comparison of ghrelin, glucose, alpha-amylase and protein levels in saliva from diabetics. BMB Rep 2007;40:29-35.

15. Zakowski JJ, Bruns DE. Biochemistry of human alpha amylase isoenzymes. CRC Crit Rev Clin Lab Sci 1985;21:283-322.

16. Bonnefond A, Yengo L, Dechaume A, et al. Relationship between salivary/pancreatic amylase and body mass index: A systems biology approach. BMC Med 2017;15:37.

17. Falchi M, Moustafa JSES, Takousis P, et al. Low copy number of the salivary amylase gene predisposes to obesity. Nat Genet 2014; 46:492-497.

18. Nakajima K, Nemoto T, Muneyuki T, et al. Low serum amylase in association with metabolic syndrome and diabetes: A communitybased study. Cardiovasc Diabetol 2011;10:34.

19. Nakajima K, Muneyuki T, Munakata H, et al. Revisiting the cardiometabolic relevance of serum amylase. BMC Res Notes 2011;4:419.

20. Mandel AL, Breslin PA. High endogenous salivary amylase activity is associated with improved glycemic homeostasis following starch ingestion in adults. J Nutr 2012;142:853-858.

21. Harthoorn LF, Dransfield E. Periprandial changes of the sympathetic-parasympathetic balance related to perceived satiety in humans. Eur J Appl Physiol 2008;102:601-608.

22. Harthoorn LF, Schipper RG, Loof A, et al. Salivary biomarkers associated with perceived satiety and body mass in humans. Proteomics Clin Appl 2007;1:1637-1650.

23. Harthoorn LF. Salivary $\alpha$-amylase: A measure associated with satiety and subsequent food intake in humans. Int Dairy J 2008;18:879-883.

24. Shabat-Simon M, Shuster A, Sela T, et al. Objective physiological measurements but not subjective reports moderate the effect of hunger on choice behavior. Front Psychol 2018;9:750.
25. Nater UM, Rohleder N, Scholotz W, et al. Determinants of the diurnal course of salivary sAA. Psychoneuroendrocrinology 2007; 32:392-401.

26. Cole TJ, Bellizzi MC, Flegal KM, et al. Establishing a standard definition for child overweight and obesity worldwide: International survey. BMJ 2000;320:1240-1245.

27. Walker RF, Riad-Fahmy D, Read GF. Adrenal status assessed by direct radioimmunoassay of cortisol in whole saliva or parotid saliva. Clin Chem 1978;24:1460-1463.

28. Walker RF, Read GF, Riad-Fahmy D. Radioimmunoassay of progesterone in saliva: Application to the assessment of ovarian function. Clin Chem 1979;25:2030-2033.

29. Sánchez-Navarro JP, Maldonado EF, Martínez-Selva JM, et al. Salivary alpha-amylase changes promoted by sustained exposure to affective pictures. Psychophysiology 2012;49:1601-1609.

30. Beltzer EK, Fortunato CK, Guaderrama MM, et al. Salivary flow and alpha-amylase: Collection technique, duration, and oral fluid type. Physiol Behav 2010;101:289-296.

31. Moreno-Padilla M, Fernández-Serrano MJ, Reyes Del Paso GA Risky decision-making after exposure to a food-choice task in excess weight adolescents: Relationships with reward-related impulsivity and hunger. PLoS One 2018;13:e0202994.

32. Benelam B. Satiation, satiety and their effects on eating behaviour. Nutr Bull 2009;34:126-173.

33. Bruce AS, Holsen LM, Chambers RJ, et al. Obese children show hyperactivation to food pictures in brain networks linked to motivation, reward and cognitive control. Int J Obes 2010;34: 1494-1500.

34. Davids S, Lauffer H, Thoms K, et al. Increased dorsolateral prefrontal cortex activation in obese children during observation of food stimuli. Int J Obes 2010;34:94-104.

35. Yokum S, Ng J, Stice E. Attentional bias to food images associated with elevated weight and future weight gain: An fMRI study. Obesity 2011;19:1775-1783.

36. Batterink L, Yokum S, Stice E. Body mass correlates inversely with inhibitory control in response to food among adolescent girls: An fMRI study. Neuroimage 2010;52:1696-1703.

37. Yokoyama J, Tajima N, Ikeda Y, et al. The amylase activity and its isoenzyme analysis in juvenile-onset diabetes mellitus. Tonyobyo 1980;23:607-617.

38. Swislocki A, Noth R, Hallstone A, et al. Triadafilopoulos G. Secretin-stimulated amylase release into blood is impaired in type 1 diabetes mellitus. Horm Metab Res 2005;37:326-330.

39. Schneiderman N, Skyler JS. Insulin metabolism, sympathetic nervous system regulation, and coronary heart disease prevention. In: Orth-Gomér K, Schneiderman N (eds), Behavioral Medicine Approaches to Cardiovascular Disease Prevention. Lawrence Erlbaum Associates, Inc.: Hillsdale, NJ, 1996. pp. 105-133.

40. Kuebler U, von Känel R, Heimgartner N, et al. Norepinephrine infusion with and without alpha-adrenergic blockade by phentolamine increases salivary alpha amylase in healthy men. Psychoneuroendocrinology 2014;49:290-298.

Address correspondence to: María Moreno-Padilla, PhD Departamento de Psicología Universidad de Jaén Jaén 23071 Spain

E-mail: maria.moreno@ujaen.es 